\title{
Dosimetric Comparisons of Lung SBRT with Multiple Metastases by Two Advanced Planning Systems
}

\author{
Ye Zhang1, Yie Chen², Jie Qiư ${ }^{3}$ Jack Yang2 \\ ${ }^{1}$ Department of Radiation Oncology, Cancer Institute and Hospital Chinese Academy Medical Sciences \\ (CICAMS), Beijing, China \\ ${ }^{2}$ Department of Radiation Oncology, Monmouth Medical Center, Long Branch, USA \\ ${ }^{3}$ Department of Radiation Oncology, Beijing Union Hospital, Beijing, China \\ Email: jyang@barnabashealth.org
}

Received 27 August 2014; revised 26 September 2014; accepted 25 October 2014

Copyright (C) 2014 by authors and Scientific Research Publishing Inc.

This work is licensed under the Creative Commons Attribution International License (CC BY). http://creativecommons.org/licenses/by/4.0/

(c) (i) Open Access

\section{Abstract}

Purpose: To evaluate planning quality of Stereotactic body Radiotherapy (SBRT) with multiple lungmetastases generated by the Pinnacle and Tomotherapy planning systems, respectively. Methods and Materials: Nine randomly selected patients diagnosed with non-small cell lung carcinoma with multiple lesions were planned with Philips Pinnacle (version 9.2, Fitchburg, WI) and Tomotherapy (version 4.2, Madison, WI), respectively. Both coplanar and non-coplanar IMRT plans were generated on Pinnacle system. A total dose of 60 Gy was prescribed to cover $95 \%$ of Planning Target Volume (PTV) in 3 fractions based on the RTOG0236 protocol prescription [1]. All plans with single isocenter setting were used for multiple lesions planning. A set of nine static beams were used for Pinnacle plansusing Direct Machine Parameters Optimization (DMPO) algorithm of RTOT0236 dose constraints. Planning outcomes such as minimum and mean doses, $\mathrm{V}_{95}$, $D_{95}\left(95 \%\right.$ of target volume receivesprescription dose), $D_{5}$, and $D_{1}$ to PTV, maximum dose to heart, esophagus, cord, trachea, brachial plexus, rib, chest wall, and liver, mean dose toliver, total lung, right and left lung, volume of chest wall receives $30 \mathrm{~Gy}$, volume of lungs receives 5 Gy and 20 Gy $\left(\mathrm{V}_{5}\right.$ and $\left.\mathrm{V}_{20}\right)$, conformity index (CI) and heterogeneity index (HI) were all reported for evaluation. Results: Mean volume of PTV was $37.77 \pm 23.4 \mathrm{~cm}^{3}$. $\mathrm{D}_{95}$ of PTV with Tomotherapy, coplanar, non-coplanar plan was $60.2 \pm 0.3 \mathrm{~Gy}, 58.6 \pm 1.2 \mathrm{~Gy}$, and $59.1 \pm 0.7 \mathrm{~Gy}$, respectively. Mean dose to PTV was lower for Tomotherapy $(p<0.0001)$, so were $D_{5}(p<0.0001)$ and $D_{1}(p=0.001)$. CI was higher with Tomotherapy plans $(p<0.0001)$, so was HI $(p<0.0001)$. Maximum dose to other critical organs were also lower exclusively with Tomotherapy plans, as expected. Treatment time was recorded only for Tomotherapy plans $(73.0 \pm 20.6 \mathrm{~min})$ while the Intensity Modulated Radiation Therapy (IMRT) plan from Pinnacle were not registered for comparison in those cases. Conclusions: With 51 beam angles per rotation, Tomotherapy plans could generally achieve better tumor coverage 
while sparing more critical structures in the multiple lung lesions study. Non-coplanar IMRT plans also have better tumor coverage with lower dose to critical organs such as lungs, liver, chest wall and cord compare to coplanar plans. Compared to the coplanar IMRT beam plans, Tomotherapy tends to have a relatively higher low dose volume in lungs such as $V_{5}$ which needs more attention for toxicity analysis.

\section{Keywords}

\section{SBRT, DMPO, Tomotherapy, Lung IMRT}

\section{Introduction}

With early stage primary non-small-cell lung cancer (NSCLC) of T1 or T2 lesion not including metastases, usually the surgical resection was chosen to manage using a lobectomy technique. Unfortunately, significant complication could be associated with lobectomy for those medically inoperable patients [2]-[3]. SBRT has been shown to be an effective treatment option for inoperable patients with lung cancer and metastatic lung lesions. Noticeable local tumor control rates had been reported with SBRT treatment technique but most reports have only one lesion in their studies [4]-[6]. However, most reports in this area documented patients with only one lung lesion instead of multiple lung lesions. Kelly et al. [7] has studied lung patients with up to three multiple metastatic lung lesions and reported no grade 4 - 5 toxicity. Another group by Okunieff et al. [8] also reported dosimetric evaluation with more than five metastatic lesions but without any explicit reports on the outcomes of the SBRT treatments. In order to acquire the most valuable dosimetric information and understand how different radiation schedules is being adopted with fractionated scheme for SBRT in lung, we have also listed the Biological Equivalent Dose (BED) derived from the report of Kavanagh et al. [9]. This simple table has not correctly predicted a linear quadratic correlation between the lung lesions and late responding normal tissues; however, it does support the concept of SBRT which could generate a higher equivalent dose for hypofractionated treatment schemes (see Table 1).

There have been reports which relate the clinical efficacy of SBRT over different fractionations to a BED cutoff. Thus, Onishi, et al. [10] reported that improved local control and survival are associated with SBRT regimens whose BED is $>100 \mathrm{~Gy}$. In other words, the purpose of SBRT treatment is to produce a higher tumor control intent and safer outcome with possible minimum complications. The recognition of dose escalation for hypofractionated treatment has gained popularity in recent years, with many clinical implementation, presented the successful local control of metastatic lung disease treatment [11]-[13].

Traditional SBRT treatment was performed with 3-D conformal therapy. The basic principle is to utilize a set of static beams and to organize the optimized beam and couch angle combinations in order to form the best dose coverage to the target. This process needs tremendous amount of experience and can be system dependent to avoid collision in case they are non-coplanar. The process could be tedious and time consuming. Using IMRT could be a more efficient way to obtain the expected dose coverage, while minimizing the normal structures, either by coplanar or non-coplanar field and beam designs. Tomotherapy is a rotational unit for helical pattern

Table 1. Biological equivalent dose (conventional scheme vs. SBRT).

\begin{tabular}{ccc}
\hline \multicolumn{2}{c|}{ Biological Equivalent Dose (BED) } & BED (Gy) \\
\hline Conventional Radiation Dose (Gy) & Fraction & 72 \\
\hline 60 & 30 & 84 \\
Total SBRT Dose (Gy) & 35 & 125 \\
48 & 3 & 132 \\
60 & 5 & 180 \\
\hline
\end{tabular}


treatment. With that, the calculated dose distribution could be utilized to compare with Tomotherapy technique.

In this study, we have specifically designed the treatment protocols to reproduce the dosimetry and targeting patients which related to the plans carried out by Pinnacle planning system, either with coplanar and non-coplanar beams entries.

\section{Method and Materials}

Nine patients diagnosed with NSCLC staged from T1 - T3 with multiple lung metastases were selected $(n=9)$ for this dosimetric study. There were two patients with two lesions on the right lung, one patient with two lesions on the left lung, five patients with one lesion on the right and one lesion on the left lung, and one patient with two lesions on the right side and one lesion on the left. The range of PTV was from 14.31 to 91.26 cc which presented the mean volume of $37.6 \pm 23.4$ cc. Plans on the Pinnacle system were programmed with nine non-opposing gantry angles (0, 30, 135, 165, 200, 240, 270, 300 and 330 degrees) for coplanar beams. And with non-coplanar beam settings, the following combination of gantry and couch angles was implemented for individual lung planning (Table 2).

The energy was selected at $6 \mathrm{MV}$ photons; the prescription was $20 \mathrm{~Gy}$ per fraction with a total dose of $60 \mathrm{~Gy}$ (3 SBRT fractions). Single isocenter on Pinnacle was set to the geometric center of the two or more lesions for planning and prescription purposes. The sorted DICOM images with isocenter and structures such as PTV, lungs (exclude GTV), heart, cord, cord-exp (1 cm expansion of contoured cord structure), trachea, brachial plexus, ribs, chest wall, high dose, low dose (external contour minus $2 \mathrm{~cm}$ expansion of PTV), RING structure (external subtract $1 \mathrm{~cm}$ of PTV expansion) were transferred from Pinnacle to Tomotherapy. The RING structure was used to avoid any hot spot and to generate a smoother dose distribution among the three planning tools. For both coplanar and non-coplanar plans, Direct Machine Parameters Optimization (DMPO) was used for optimization, max iterations was 50 , convolution dose iteration was 20 , maximum number of segments were set to 50 which was tested to suit most of the clinical cases, minimum segment area was $4 \mathrm{~cm}^{2}$, and minimum segment MUs was designed 5 to avoid small MU delivery. For Tomotherapy plans, the jaw width was set at $1 \mathrm{~cm}$, grid size was set to normal, modulation factor was preset at 1.7 for four cases, and five cases were programmed at 1.4 due to problem with gantry period (Tomotherapy has the speed limit with maximum 6 rotations in 1 minute). Pitch was 0.09 for one case, and other eight cases were set to 0.1. Similar optimization goals were set for all cases. Multivariate analysis using PASW ${ }^{\mathrm{TM}}$ (formerly SPSS ${ }^{\mathrm{TM}}$ ) statistical tool version 18 with a significance level of 0.05 were used for statistical analysis (i.e. $p<0.05$ indicates clinical significance among the study cases).

Planning outcomes such as $\mathrm{V}_{95}$ (95\% prescribed dose to volume, $\mathrm{D}_{99}$ (99\% of the target volume receives a minimum of $90 \%$ of the prescription dose), $\mathrm{D}_{95}$ ( $95 \%$ of the target volume receives the prescribed dose), $\mathrm{D}_{5}, \mathrm{D}_{1}$, mean and minimum dose to PTV; $\mathrm{V}_{20}$ (percent volume receiving $20 \mathrm{~Gy}$ ), $\mathrm{V}_{5}$ (percent volume receiving $5 \mathrm{~Gy}$ ), and mean dose to lungs; Maximum dose to heart, esophagus, cord, trachea, brachial plexus, rib, and chest wall; $\mathrm{V}_{30}$ (percent volume receiving $30 \mathrm{~Gy}$ ) to chest wall, and mean dose to liver were reported for this study.

Table 2. Gantry and couch combination for non-coplanar beam settings.

\begin{tabular}{|c|c|c|c|c|c|}
\hline \multirow{2}{*}{$\begin{array}{c}\text { Right Lung Lesion } \\
\text { (IEC Scale) }\end{array}$} & \multicolumn{2}{|c|}{ Degrees } & \multirow{2}{*}{$\begin{array}{c}\text { Left Lung Lesion } \\
\text { (IEC Scale) }\end{array}$} & \multicolumn{2}{|c|}{ Degrees } \\
\hline & Gantry & Couch & & Gantry & Couch \\
\hline & 180 & 0 & & 180 & 0 \\
\hline & 220 & 20 & & 210 & 0 \\
\hline & 270 & 335 & & 270 & 0 \\
\hline & 270 & 25 & & 315 & 25 \\
\hline & 315 & 0 & & 30 & 90 \\
\hline & 30 & 90 & & 330 & 90 \\
\hline & 330 & 90 & & 90 & 25 \\
\hline & 45 & 335 & & 90 & 335 \\
\hline & 90 & 0 & & 160 & 340 \\
\hline
\end{tabular}


$\mathrm{V}_{105}$ (high dose) was $105 \%$ of prescribed dose delivered to volume of body minus PTV, low dose as the falloff gradient was defined as dose to volume of body minus $2 \mathrm{~cm}$ expansion of PTV according to RTOG0236 protocol [1].

$\mathrm{CI}$ and $\mathrm{HI}$ were also evaluated according to RTOG0236 protocol as:

$$
\mathrm{CI}=\mathrm{V}_{\mathrm{PIV}} / \mathrm{V}_{\mathrm{PTV}}
$$

where $\mathrm{V}_{\mathrm{PTV}}$ is the volume of PTV, and $\mathrm{V}_{\mathrm{PIV}}$ is volume of prescription isodose volume. The ideal $\mathrm{CI}$ is $<1.2$ with a minimum tumor size of $3.5 \mathrm{~cm}$.

HI was also defined by RTOG0236protocol as:

$$
\mathrm{HI}=\mathrm{D}_{5} / \mathrm{D}_{95}
$$

where $\mathrm{D}_{5}$ is prescribed dose to cover $5 \%$ of PTV, and $\mathrm{D}_{95}$ is prescribed dose to cover $95 \%$ of PTV.

\section{Results}

Treatment plans were executed on the Pinnacle system by setting the same dose constraints as in Tomotherapy with the same contours transferred from Pinnacle. The coplanar was with 40 degrees separation each, and the non-coplanar beams were followed with the same angles but with couch rotations in a pre-set parameters for left and right lung lesions, respectively.

\subsection{CI}

The ranges of CI for Tomotherapy, Pinnacle coplanar and non-coplanar were from 1.01 to 1.07, 0.67 to 0.95 , and 0.73 to 1.01 , respectively. A statistical significance was observed compared Tomotherapy to non-coplanarbema settings $(p<0.0001)$ and coplanar $(p<0.0001)$.

\subsection{HI}

The ranges of HI for Tomotherapy, Pinnacle coplanar and non-coplanar were from 1.02 to 1.05, 1.07 to 1.14, and 1.05 to 1.12 , respectively.

A statistical significance was observed compare between Tomotherapy and non-coplanar $(p<0.0001)$, noncoplanar and coplanar $(p<0.0001)$ (Table 3).

\subsection{0\% of Prescription Volume to the PTV (R50\%)}

RTOG0236 asked to report the R50\% values, the ranges of ratios of 50\% prescription isodose volume to PTV for Tomotherapy, Pinnacle coplanar and non-coplanar were from 2.32 to $9.11 \mathrm{~Gy}, 7.45$ to $14.03 \mathrm{~Gy}$, and 6.98 to $10.71 \mathrm{~Gy}$, respectively. A statistical significance was observed compare Tomotherapy to non-coplanar $(p=$ $0.004)$ and coplanar $(p<0.0001)$ (Figure 1).

\subsection{PTV}

The ranges of mean dose to PTV for Tomotherapy, coplanar and non-coplanar were from 60.64 to 61.99 Gy, 61.84 to 61.88 , and 61.85 to 61.86 Gy, respectively. A statistical significance was observed compare Tomotherapy with non-coplanar $(p<0.0001)$ and coplanar $(p<0.0001)$. The ranges of minimum dose to PTV for Tomotherapy, coplanar and non-coplanar were from 55.65 to $58.42 \mathrm{~Gy}, 5344$ to $56.09 \mathrm{~Gy}$, and 50.50 to $55.88 \mathrm{~Gy}$, respectively.

The ranges of minimum dose to PTV for Tomotherapy, coplanar and non-coplanar were from 55.65 to 58.42

Table 3. Planning results of CI and HI.

\begin{tabular}{ccc}
\hline & CI & HI \\
\hline Tomotherapy & $1.04 \pm 0.02$ & $1.03 \pm 0.01$ \\
Coplanar & $0.83 \pm 0.09$ & $1.09 \pm 0.03$ \\
Non-coplanar & $0.88 \pm 0.09$ & $1.08 \pm 0.02$ \\
\hline
\end{tabular}




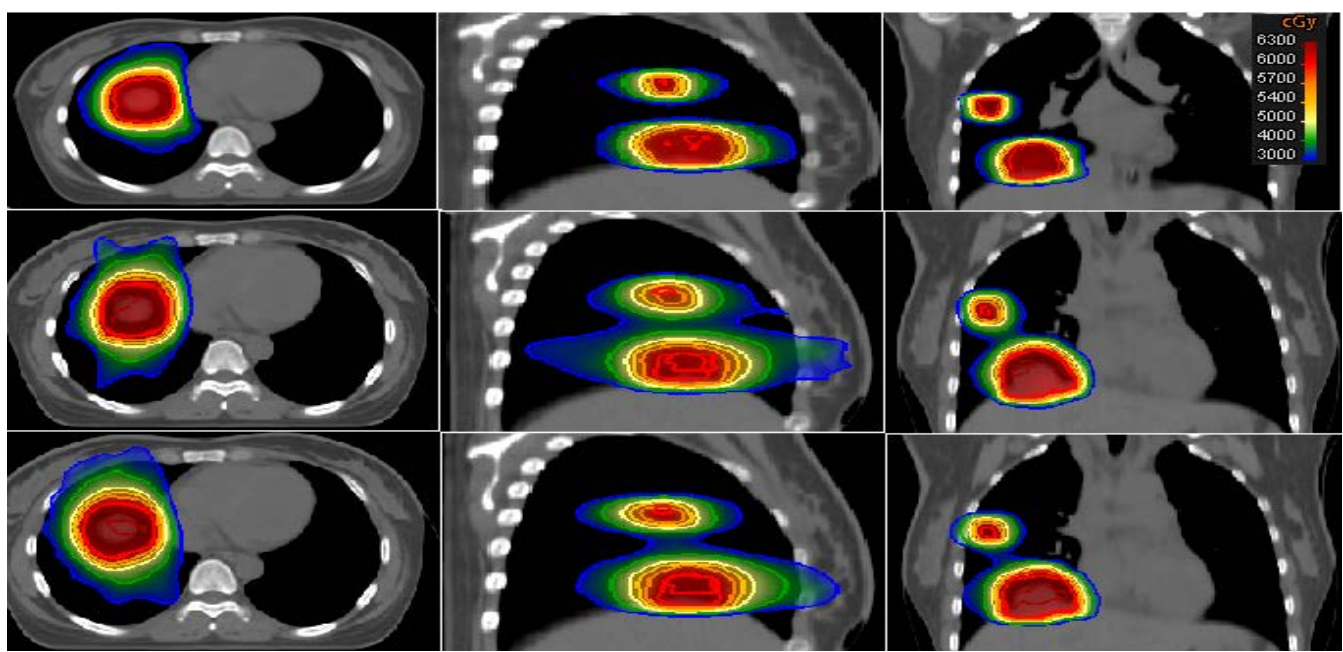

Figure 1. Isodose of one patient on Tomotherapy (top), Non-coplanar (middle), and Coplanar (bottom) in axial, sagittal and coronal planes.

Gy, 5344 to 56.09 Gy, and 50.50 to 55.88 Gy, respectively. The ranges of mean dose to PTV for Tomotherapy, coplanar and non-coplanar were from 60.69 to $61.99 \mathrm{~Gy}, 61.84$ to $61.86 \mathrm{~Gy}$, and 61.84 to $61.86 \mathrm{~Gy}$, respectively.

The ranges of $\mathrm{V}_{95}$ for Tomotherapy, coplanar and non-coplanar were from $96.1 \%$ to $100 \%$, $92.88 \%$ to $100 \%$, and $93.68 \%$ to $100 \%$, respectively. The ranges $\mathrm{D}_{5}$ for Tomotherapy, coplanar and non-coplanar were from 61.5 to $63.4 \mathrm{~Gy}, 62.88$ to $65 \mathrm{~Gy}$, and 62.92 to $64.79 \mathrm{~Gy}$, respectively. Significance was observed compare Tomotherapy to non-coplanar $(p<0.0001)$ and coplanar $(p<0.0001)$.

The ranges of $D_{1}$ for Tomotherapy, coplanar and non-coplanar were from 61.52 to 63.76 Gy, 63.19 to 65.54 Gy, and 63.84 to $65.87 \mathrm{~Gy}$, respectively. A statistical significance was also observed compare Tomotherapy and non-coplanar $(p=0.001)$ and coplanar $(p=0.001)$ (Table 4$)$.

\subsection{High Dose Area}

The volume of high dose receiving $105 \%$ of prescribed dose for Tomotherapy, Pinnacle coplanar and non_coplanar was from $0 \%, 0 \%$ to $35.59 \%$, and $0 \%$ to $15.96 \%$, respectively. The dose greater than $105 \%$ of the prescription dose occurred primarily within the PTV.

\subsection{Low Dose Area}

The ranges of maximum dose to low dose for Tomotherapy, Pinnacle coplanar and non-coplanar were from 27.92 to $42.68 \mathrm{~Gy}, 34.66$ to $45.69 \mathrm{~Gy}$, and 37.35 to $49.33 \mathrm{~Gy}$, respectively.

A statistical significance was observed compare Tomotherapy to non-coplanar $(p=0.004)$ and coplanar $(p=$ $0.001)$.

\subsection{Total Lung}

The ranges of mean dose to total lung for Tomotherapy, Pinnacle coplanar and non-coplanar were from 1.12 to $11.68 \mathrm{~Gy}, 6.09$ to $13.17 \mathrm{~Gy}$, and 6.35 to $14.33 \mathrm{~Gy}$, respectively. The volume of total lung receiving $20 \mathrm{~Gy}$ for Tomotherapy, Pinnacle coplanar and non-coplanar were from $5.27 \%$ to $23.71 \%, 7.31 \%$ to $23.99 \%$, and $7.23 \%$ to $24.21 \%$, respectively. The volume of total lung receiving 5 Gy for Tomotherapy, Pinnacle coplanar and noncoplanar were from $28.67 \%$ to $61.93 \%$, $25.58 \%$ to $63.89 \%$, and $33.57 \%$ to $76.93 \%$, respectively (Table 5).

\subsection{Right Lung}

The ranges of mean dose to right lung for Tomotherapy, Pinnacle coplanar and non-coplanar were from 1.07 to $16.85 \mathrm{~Gy}, 3.4$ to $19.82 \mathrm{~Gy}$, and 2.85 to $20.05 \mathrm{~Gy}$, respectively. The volume of right lung receiving $20 \mathrm{~Gy}$ for 
Table 4. PTV coverage statistics among three planning methodologies.

\begin{tabular}{ccccccc}
\hline & \multicolumn{9}{c}{ PTV (Gy) } & \multicolumn{2}{c}{ PTV (\%) } \\
\hline & $\mathrm{D}_{95}$ & $\mathrm{D}_{5}$ & $\mathrm{D}_{1}$ & $\mathrm{D}_{\min }$ & $\mathrm{D}_{\text {mean }}$ & $\mathrm{V}_{95}$ \\
\hline Tomotherapy & $60.2 \pm 0.3$ & $62.2 \pm 0.7$ & $62.7 \pm 0.9$ & $57.3 \pm 0.9$ & $61.2 \pm 0.4$ & $99.1 \pm 1.64$ \\
Coplanar & $58.6 \pm 1.2$ & $63.8 \pm 0.7$ & $64.4 \pm 0.7$ & $54.6 \pm 1.0$ & $61.9 \pm 0.01$ & $97.0 \pm 3.00$ \\
Non-coplanar & $59.1 \pm 0.7$ & $63.7 \pm 0.6$ & $64.4 \pm 0.8$ & $54.0 \pm 1.7$ & $61.9 \pm 0.007$ & $97.6 \pm 2.53$ \\
\hline
\end{tabular}

Table 5. Total lung volume statics among three planning methodologies.

\begin{tabular}{cccc} 
& & Total Lung & \\
\cline { 2 - 4 } & $\mathrm{V}_{20}(\%)$ & $\mathrm{D}_{\text {mean }}(\mathrm{Gy})$ & $\mathrm{V}_{5}(\%)$ \\
\hline Tomotherapy & $13.7 \pm 6.8$ & $7.8 \pm 3.6$ & $42.6 \pm 10.4$ \\
Coplanar & $16.9 \pm 6.7$ & $9.3 \pm 2.8$ & $39.9 \pm 11.3$ \\
Non-coplanar & $17.0 \pm 7.9$ & $9.5 \pm 2.9$ & $45.4 \pm 13.3$ \\
\hline
\end{tabular}

Tomotherapy, Pinnacle coplanar and non-coplanar were from $0 \%$ to $34.87 \%$, $0.03 \%$ to $44.84 \%$, and $0 \%$ to 45.25\%, respectively. The volume of right lung receiving 5 Gy for Tomotherapy, Pinnacle coplanar and noncoplanar were from $24.14 \%$ to $70.05 \%$, $22.68 \%$ to $72.42 \%$, and $16.62 \%$ to $77.34 \%$, respectively (Table 6 ).

\subsection{Left Lung}

The ranges of mean dose to left lung for Tomotherapy, Pinnacle coplanar and non-coplanar were from 1.56 to 11.45 Gy, 1.6 to $14.07 \mathrm{~Gy}$, and 1.31 to $14.41 \mathrm{~Gy}$, respectively. The volume of left lung receiving 20 Gy for Tomotherapy, Pinnacle coplanar and non-coplanar were from $0 \%$ to $21.68 \%$, $0 \%$ to $31.09 \%$, and $0 \%$ to $27.98 \%$, respectively. The volume of left lung receiving $5 \mathrm{~Gy}$ for Tomotherapy, Pinnacle coplanar and non-coplanar were from $32.72 \%$ to $48.59 \%, 7.62 \%$ to $51.13 \%$, and $0.81 \%$ to $63.09 \%$, respectively (Table 7 ).

\subsection{Heart}

The ranges of maximum dose to heart for Tomotherapy, Pinnacle coplanar and non-coplanar were from 1.26 to 64.91 Gy, 1.26 to $61.72 \mathrm{~Gy}$, and 16.85 to $61.72 \mathrm{~Gy}$.

\subsection{Esophagus}

The ranges of maximum dose to esophagus for Tomotherapy, Pinnacle coplanar and non-coplanar were from 0.65 to $33.27 \mathrm{~Gy}, 0.44$ to $26.17 \mathrm{~Gy}$, and 5.22 to $29.23 \mathrm{~Gy}$.

\subsection{Cord}

The ranges of maximum dose to cord for Tomotherapy, Pinnacle coplanar and non-coplanar were from 6.72 to 23.85 Gy, 14.43 to $32.33 \mathrm{~Gy}$, and 12.79 to $29.55 \mathrm{~Gy}$.

\subsection{Trachea}

The ranges of maximum dose to trachea for Tomotherapy, Pinnacle coplanar and non-coplanar were from 8.81 to $30.18 \mathrm{~Gy}, 9.86$ to $37.52 \mathrm{~Gy}$, and 8.45 to $39.34 \mathrm{~Gy}$.

\subsection{Brachial Plexus}

Only two cases were contoured and evaluated due to its proximity location. The ranges of brachial plexus for Tomotherapy, Pinnacle coplanar and non-coplanar were from 8.80 to 23.26 Gy, 11.41 to 27.71 Gy, and 29.73 to 33.46 Gy, respectively (Table 8). 
Table 6. Individual lung (right) volume statistics among three planning methodologies.

\begin{tabular}{cccc}
\hline & & Right Lung & \\
\cline { 2 - 4 } & $\mathrm{V}_{20}(\%)$ & $\mathrm{D}_{\text {mean }}(\mathrm{Gy})$ & $\mathrm{V}_{5}(\%)$ \\
\hline Tomotherapy & $14.93 \pm 12.8$ & $7.9 \pm 5.4$ & $41.6 \pm 15.5$ \\
Coplanar & $17.9 \pm 14.6$ & $9.6 \pm 5.5$ & $40.9 \pm 18.4$ \\
Non-coplanar & $18.2 \pm 15.3$ & $10.0 \pm 5.8$ & $44.8 \pm 21.0$ \\
\hline
\end{tabular}

Table 7. Individual lung (left) volume statistics among three planning methodologies.

\begin{tabular}{cccc}
\hline & & Left Lung & \\
\cline { 2 - 4 } & $\mathrm{V}_{20}(\%)$ & $\mathrm{D}_{\text {mean }}(\mathrm{Gy})$ & $\mathrm{V}_{5}(\%)$ \\
\hline Tomotherapy & $0.11 \pm 0.08$ & $7.6 \pm 3.5$ & $43.5 \pm 10.8$ \\
Coplanar & $0.14 \pm 0.08$ & $8.4 \pm 4.1$ & $38.4 \pm 19.9$ \\
Non-coplanar & $0.13 \pm 0.08$ & $8.4 \pm 4.4$ & $42.4 \pm 25.4$ \\
\hline
\end{tabular}

Table 8. Critical organ volume statistics among three planning methodologies.

\begin{tabular}{|c|c|c|c|c|c|}
\hline & Heart & Esophagus & Cord & Trachea & Brachial Plexus \\
\hline & $\mathrm{D}_{\max }$ & $\mathrm{D}_{\max }$ & $\mathrm{D}_{\max }$ & $\mathrm{D}_{\max }$ & $\mathrm{D}_{\max }$ \\
\hline Tomotherapy & $32.0 \pm 18.5$ & $16.5 \pm 8.7$ & $15.6 \pm 4.5$ & $16.2 \pm 9.8$ & $3.6 \pm 7.9$ \\
\hline Coplanar & $36.1 \pm 20.6$ & $18.7 \pm 8.2$ & $19.3 \pm 5.8$ & $18.8 \pm 11.4$ & $4.3 \pm 9.5$ \\
\hline Non-coplanar & $39.1 \pm 15.8$ & $16.6 \pm 6.6$ & $17.4 \pm 7.9$ & $19.7 \pm 11.8$ & $7.0 \pm 14.0$ \\
\hline
\end{tabular}

\subsection{Ribs}

The ranges of maximum dose to ribs for Tomotherapy, Pinnacle coplanar and non-coplanar were from 31.97 to 62.3 Gy, 39.32 to 65.55 Gy, and 45.98 to 66.59 Gy, respectively.

\subsection{Chest Wall}

The ranges of maximum dose to chest wall for Tomotherapy, coplanar and non-coplanar were from 39.28 to 63.46 Gy, 44.39 to $64.09 \mathrm{~Gy}$, and 49.21 to $65.06 \mathrm{~Gy}$, respectively. The volume of chest wall receiving dose of 30 Gy with Tomotherapy, coplanar and non-coplanar were from $0.19 \%$ to $9.28 \%$, $0.64 \%$ to $13.52 \%$, and $1.94 \%$ to $11.96 \%$, respectively.

\subsection{Liver}

The ranges of mean dose to liver for Tomotherapy, Coplanar, and Non-coplanar were from 1.6 to $12.06 \mathrm{~Gy}, 1.00$ to $8.80 \mathrm{~Gy}$, and 3.00 to $14.92 \mathrm{~Gy}$, respectively (Table 9).

\subsection{Monitor Units and Treatment Time}

The monitor units for Coplanar and Non-coplanar were evaluated. The ranges for Coplanar and Non-coplanar were from 4527 to $8750 \mathrm{MU}$, and 4967 to $9081 \mathrm{MU}$, respectively. The treatment time for Tomotherapy was recorded; the range was from 44.6 to 100.5 min.; the least time was from both lesions on the left, and the most time consuming part was from the one with three lesions (two located on the right and one on the left).

\section{Discussion}

SBRT treatment opens a new era for treating the lung metastases compared to the conventional surgery, which was invasive with higher risks. In North America, SBRT has been the standard choice of treatment for selected 
Table 9. List of doe statistics of three critical structures.

\begin{tabular}{|c|c|c|c|c|}
\hline & Rib & \multicolumn{2}{|c|}{ Chest Wall } & Liver \\
\hline & $\mathrm{D}_{\max }(\mathrm{Gy})$ & $\mathrm{D}_{\max }(\mathrm{Gy})$ & $\mathrm{V}_{30}(\%)$ & $\mathrm{D}_{\text {mean }}(\mathrm{Gy})$ \\
\hline Tomotherapy & $54.9 \pm 10.6$ & $57.5 \pm 8.1$ & $4.73 \pm 3.46$ & $2.24 \pm 3.9$ \\
\hline Coplanar & $59.4 \pm 8.2$ & $60.1 \pm 6.8$ & $8.28 \pm 4.49$ & $2.10 \pm 3.0$ \\
\hline Non-coplanar & $59.5 \pm 7.0$ & $60.4 \pm 6.2$ & $6.50 \pm 3.54$ & $2.98 \pm 4.8$ \\
\hline
\end{tabular}

group of patients with superior clinical outcome [14]. Systematic study has shown that SBRT created very comparable results to conventional approach [15]. Dunlap et al. [16] published a comparison of T1 and T2 peripheral lesion SBRT treatment comparison, with emphasis on the tumor size factor based on the Tomotherapy delivery results. The corresponding CI and HI were 1.17 and 1.06, respectively. The societies of ASTRO and AAPM have provided user guidelines of valuable information about how to safely utilizing the imaging technique as well as SBRT clinical practices [17]-[19]. As cross checked and examined in the literatures [20]-[23], patients with multiple lung metastases presented dosimetric challenges in 3D conformal therapy due to the complexity in designing the treatment ports and to avoid crossing the beam angles for hot spots. With IMRT technique, the plan might generate better coverage and eliminate possible dose spreading to the normal lung tissues. Though chemotherapy maybe an option, but the outcomes are always disappointing in managing those cases [24]. With the fast developments in radiotherapy treatment hardware and software during the last decade, two techniques have become available for the treatment of patients with multiple metastases by using static beam IMRT and/or Volumetric Modulated Arc Therapy (VMAT) technology [25]. The study by Sterzing et al. [26] has already shown that helical Tomotherapy is capable of treating multiple lesions in their early investigation. Recently arc therapy treatment modality has been adopted in clinics to manage multiple lung lesions, Li et al. [27] has studied a frameless SBRT treatment with arc based planning and delivery, which reported the CI and HI with the ranges of $(0.513-0.562)$ and $(0.0709-0.0794)$ of two plans. However, the CI and HI indexes were not derived from the RTOG0236 protocol, but they have indicated the $\mathrm{V}_{20}$ values fell into $8.46 \%$ and $14.39 \%$, respectively. They were utilizing the Elekta ${ }^{\mathrm{TM}}$ system VMAT technique to compare the planning results with multiple lung lesions. This article also demonstrated the arc based therapy could be beneficial to create suitable dose distribution with reasonable sparing to normal lung tissues. Monte Carlo calculation also has been proposed to avoid and dose discrepancy in the lung-tissue interface for inhomogeneity corrections. Lax et al. [28] has found that the results of their particular study showed average differences of $9 \%$ (minimum, $-8 \%$; maximum, 29\%), 12\% (minimum, 0; maximum, 28\%), 7\% (minimum, 3\%; maximum, 13\%), and 18\% (minimum, 11\%; maximum, 29\%) in $R_{100 \%}, R_{50 \%}, D_{2} \mathrm{~cm}$, and $V_{20}$, respectively. However, the current calculation algorithm in our study was still based on convolution/superposition which Tomotherapy and Pinnacle systems utilized in RTOG2036 comparisons.

The planning comparison focused on the physics strength from helical delivery, along with simple steps in creating an acceptable plan with better planning quality. Helical Tomotherapy presented with a good option to plan and treat those tough cases (multiple lesions) with very encouraging clinical outcomes. Our study has shown that Tomotherapy has better coverage and less normal tissues doses among those 9 patients, as they only concentrate on the feasibility of planning multiple lung lesions with one isocenter. The treatment time was not compared against coplanar or non-coplanar plans from Pinnacle systems due to the continuous couch movement with Tomotherapy delivery, which the MLC was in fact close in between PTVs. The Tomotherapy delivery time was be longer than the static beams treatment in IMRT. Though TomoEdge ${ }^{\mathrm{TM}}$ has been released for clinical usage, but at this moment, our system was not upgraded to execute this option, which can dramatically reduce the treatment delivery time by varying the jaw sizes to reduce the time in between PTVs.

We also noticed that for the total lung planning results, where the $V_{5}$ of coplanar plan was the smallest among all the calculated results. One of the reasons was that the size of lung lesions only occupied a small portion within the lung volume, and the coplanar beams would just penetrate through the section of slices where the PTVs possessed. The static beam IMRT delivery tended to minimize the lung doses, as expected, but the tradeoff is losing coverage to cover 95\% of PTVs, which Tomotherapy had the highest scores. With all factors considered, Tomotherapy has the overall benefits of better CI and HI, and with less critical structure impact. 


\section{Conclusion}

SBRT using a helical Tomotherapy delivery unit is a well-tolerated and documented methodology in treating multiple lung metastases of inoperable early-stage NSCLC. From the conventional planning of view, it is always difficult to carry multiple areas treatments which were complex and hard to normalize. Based on our study results, dosimetric analysis of multiple lung lesions has shown that Tomotherapy could still produce higher CI and HI in selected cases. Though the cases were not treated with Linac based arc therapy technique (or VMAT) due to the availability at our center, even compared to the non-coplanar SBRT in the analysis, we found the Tomotherapy tended to create more suitable target and critical structures dose statistics as a possible factor for complications indicated [29]. However, the low dose regions created by Tomotherapy needs to be addressed more due to the spillage characteristics and the volume effect of low dose with large volume coverage may be a critical factor in toxicity analysis for future follow-up of multiple lung metastases patients.

\section{Conflict of Interests}

None.

\section{References}

[1] Timmerman, R.D., Michalski, J., Fowler, J., et al. (2008) A Phase II Trial of Stereotactic Body Radiation Therapy (SBRT) in the Treatment of Patients with Medically Inoperable Stage I/II Non-Small Cell Lung Cancer, Protocol 0236. RTOG, Philadelphia. http://www.rtog.org/members/protocols/0236/0236.pdf

[2] Pastorino, U., Buyse, M., Friedel, G., Ginsberg, R.J., Girard, P., Goldstraw, P. and Putnam Jr., J.B. (1997) Long-Term Results of Lung Metastasectomy: Prognostic analyses Based on 5206 Cases. The Journal of Thoracic and Cardiovascular Surgery, 113, 37-49. http://dx.doi.org/10.1016/S0022-5223(97)70397-0

[3] Sternberg, D.I. and Sonett, J.R. (2007) Surgical Therapy of Lung Metastases. Seminars in Oncology, 34, 186-196. http://dx.doi.org/10.1053/j.seminoncol.2007.03.004

[4] Iyengar, P. and Timmerman, R.D. (2012) Stereotactic Ablative Radiotherapy for Non-Small Cell Lung Cancer: Rationale and Outcomes. Journal of the National Comprehensive Cancer Network, 10, 1514-1520.

[5] Timmerman, R. (2010) Stereotactic Body Radiation Therapy for Inoperable Early Stage Lung Cancer. JAMA, 303, 1070-1076. http://dx.doi.org/10.1001/jama.2010.261

[6] Weichselbaum, R.R. and Hellman, S. (2011) Oligometastases Revisited. Nature Reviews Clinical Oncology, 8, 378382.

[7] Kelly, P., Balter, P. A., Rebueno, N., Sharp, H. J., Liao, Z., Komaki, R. and Chang, J. Y. (2010) Stereotactic Body Radiation Therapy for Patients with Lung Cancer Previously Treated with Thoracic Radiation. International Journal of Radiation Oncology, Biology, Physics, 78, 1387-1393. http://dx.doi.org/10.1016/j.ijrobp.2009.09.070

[8] Okunieff, P., Petersen, A.L., Philip, A., Milano, M.T., Katz, A.W., Boros, L. and Schell, M.C. (2006) Stereotactic Body Radiation Therapy (SBRT) for Lung Metastases. Acta Oncologica, 45, 808-817. http://dx.doi.org/10.1080/02841860600908954

[9] Kavanagh, B.D., McGarry, R.C. and Timmerman, R.D. (2006) Extracranial Radiosurgery (Stereotactic Body Radiation Therapy) for Oligometastases. Seminars in Radiation Oncology, 16, 77-84. http://dx.doi.org/10.1016/j.semradonc.2005.12.003

[10] Onishi, H., Shirato, H., Nagata, Y., Hiraoka, M., Fujino, M., Gomi, K. and Araki, T. (2007) Hypofractionated Stereotactic Radiotherapy (HypoFXSRT) for Stage I Non-Small Cell Lung Cancer: Updated Results of 257 Patients in Japanese Multi-Institutional Study. Journal of Thoracic Oncology, 2, S94-S100. http://dx.doi.org/10.1097/JTO.0b013e318074de34

[11] Timmerman, R.D., Bizekis, C.S., Pass, H.I., Fong, Y., Dupuy, D.E., Dawson, L.A., et al. (2009) Local Surgical, Ablative, and Radiation Treatment of Metastases. CA: A Cancer Journal for Clinicians, 59, 145-170. http://dx.doi.org/10.3322/caac.20013

[12] Rusthoven, K.E., Kavanagh, B.D., Burri, S.H., Chen, C., Cardenes, H., Chidel, M.A., et al. (2009) Multi-Institutional Phase I/II Trial of Stereotactic Body Radiation Therapy for Lung Metastases. Journal of Clinical Oncology, 27, 15791584. http://dx.doi.org/10.1200/JCO.2008.19.6386

[13] Fong, Y., Fortner, J., Sun, R.L., Brennan, M.F. and Blumgart, L.H. (1999) Clinical Score for Predicting Recurrence after Hepatic Resection for Metastatic Colorectal Cancer: Analysis of 1001 Consecutive Cases. Annals of Surgery, 230, 309. http://dx.doi.org/10.1097/00000658-199909000-00004 
[14] Timmerman, R.D., Park, C. and Kavanagh, B.D. (2007) The North American Experience with Stereotactic Body Radiation Therapy in Non-Small Cell Lung Cancer. Journal of Thoracic Oncology, 2, S101-S112. http://dx.doi.org/10.1097/JTO.0b013e318074e4fa

[15] Soldà, F., Lodge, M., Ashley, S., Whitington, A., Goldstraw, P. and Brada, M. (2013) Stereotactic Radiotherapy (SABR) for the Treatment of Primary Non-Small Cell Lung Cancer; Systematic Review and Comparison with a Surgical Cohort. Radiotherapy and Oncology, 109, 1-7. http://dx.doi.org/10.1016/j.radonc.2013.09.006

[16] Dunlap, N.E., Larner, J.M., Read, P.W., Kozower, B.D., Lau, C.L., Sheng, K., et al. (2010) Size Matters: A Comparison of T1 and T2 Peripheral Non-Small-Cell Lung Cancers Treated with Stereotactic Body Radiation Therapy (SBRT). The Journal of Thoracic and Cardiovascular Surgery, 140, 583-589. http://dx.doi.org/10.1016/j.jtcvs.2010.01.046

[17] Hartford, A.C., Galvin, J.M., Beyer, D.C., Eichler, T.J., Ibbott, G.S., Kavanagh, B., et al. (2012) American College of Radiology (ACR) and American Society for Radiation Oncology (ASTRO) Practice Guideline for Intensity-Modulated Radiation Therapy (IMRT). American Journal of Clinical Oncology, 35, 612-617. http://dx.doi.org/10.1097/COC.0b013e31826e0515

[18] Potters, L., Gaspar, L.E., Kavanagh, B., Galvin, J.M., Hartford, A.C., Hevezi, J.M., et al. (2010) American Society for Therapeutic Radiology and Oncology (ASTRO) and American College of Radiology (ACR) Practice Guidelines for Image-Guided Radiation Therapy (IGRT). International Journal of Radiation Oncology, Biology, Physics, 76, 319-325. http://dx.doi.org/10.1016/j.ijrobp.2009.09.041

[19] Potters, L., Kavanagh, B., Galvin, J.M., Hevezi, J.M., Janjan, N.A., Larson, D.A., et al. (2010) American Society for Therapeutic Radiology and Oncology (ASTRO) and American College of Radiology (ACR) Practice Guideline for the Performance of Stereotactic Body Radiation Therapy. International Journal of Radiation Oncology, Biology, Physics, 76, 326-332. http://dx.doi.org/10.1016/j.ijrobp.2009.09.042

[20] Solberg, T.D., Balter, J.M., Benedict, S.H., Fraass, B.A., Kavanagh, B., Miyamoto, C. and Yamada, Y. (2012) Quality and Safety Considerations in Stereotactic Radiosurgery and Stereotactic Body Radiation Therapy: Executive Summary. Practical Radiation Oncology, 2, 2.

[21] Lo, S.S., Fakiris, A.J., Chang, E.L., Mayr, N.A., Wang, J.Z., Papiez, L., et al. (2009) Stereotactic Body Radiation Therapy: A Novel Treatment Modality. Nature Reviews Clinical Oncology, 7, 44-54. http://dx.doi.org/10.1038/nrclinonc.2009.188

[22] Nagata, Y., Takayama, K., Matsuo, Y., Norihisa, Y., Mizowaki, T., Sakamoto, T., et al. (2005) Clinical Outcomes of a Phase I/II Study of 48 Gy of Stereotactic Body Radiotherapy in 4 Fractions for Primary Lung Cancer Using a Stereotactic Body Frame. International Journal of Radiation Oncology, Biology, Physics, 63, 1427-1431. http://dx.doi.org/10.1016/j.ijrobp.2005.05.034

[23] Milano, M.T., Katz, A.W., Muhs, A.G., Philip, A., Buchholz, D.J., Schell, M.C. and Okunieff, P. (2008) A Prospective Pilot Study of Curative-Intent Stereotactic Body Radiation Therapy in Patients with 5 or Fewer Oligometastatic Lesions. Cancer, 112, 650-658. http://dx.doi.org/10.1002/cncr.23209

[24] Siva, S., MacManus, M. and Ball, D. (2010) Stereotactic Radiotherapy for Pulmonary Oligometastases: A Systematic Review. Journal of Thoracic Oncology, 5, 1091-1099.

[25] Halpern, E.C., Perez, C.A. and Brady, L.W. (2008) Perez and Brady’s Principle and Practice of Radiation Oncology. 5th Edition, Lippincott Williams and Wilkins, Philadelphia.

[26] Sterzing, F., Welzel, T., Sroka-Perez, G., Schubert, K., Debus, J. and Herfarth, K.K. (2009) Reirradiation of Multiple Brain Metastases with Helical Tomotherapy. Strahlentherapie und Onkologie, 185, 89-93. http://dx.doi.org/10.1080/02841860600900050

[27] Li, Q., Mu, J., Gu, W., Chen, Y., Ning, Z., Jin, J. and Pei, H. (2014) Frameless Stereotactic Body Radiation Therapy for Multiple Lung Metastases. Journal of Applied Clinical Medical Physics, 15, 105-116.

[28] Lax, I., Panettieri, V. and Wennberg, B. (2006) Dose Distributions in SBRT of Lung Tumors: Comparison between Two Different Treatment Planning Algorithms and Monte-Carlo Simulation Including Breathing Motions. Acta Oncologica, 45, 978-988. http://dx.doi.org/10.1080/02841860600900050

[29] Kim, Y., Hong, S.E., Kong, M. and Choi, J. (2013) Predictive Factors for Radiation Pneumonitis in Lung Cancer Treated with Helical Tomotherapy. Cancer Research and Treatment, 45, 295-302. http://dx.doi.org/10.4143/crt.2013.45.4.295 
Scientific Research Publishing (SCIRP) is one of the largest Open Access journal publishers. It is currently publishing more than 200 open access, online, peer-reviewed journals covering a wide range of academic disciplines. SCIRP serves the worldwide academic communities and contributes to the progress and application of science with its publication.

Other selected journals from SCIRP are listed as below. Submit your manuscript to us via either submit@scirp.org or Online Submission Portal.
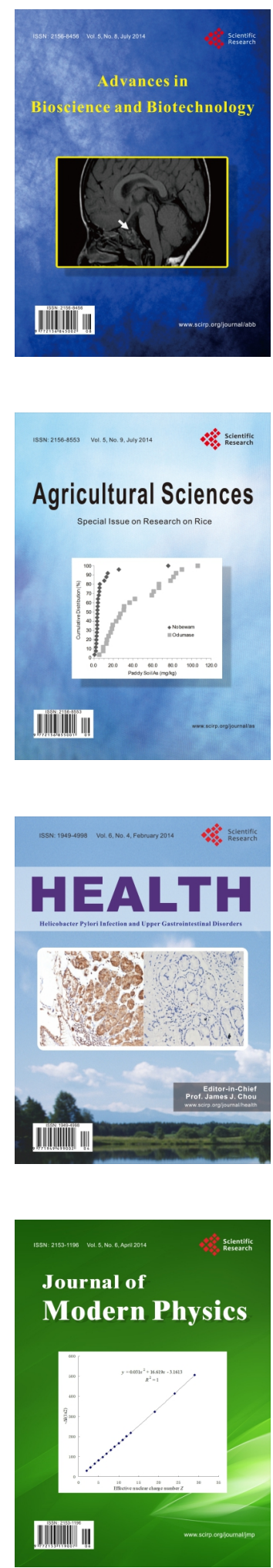
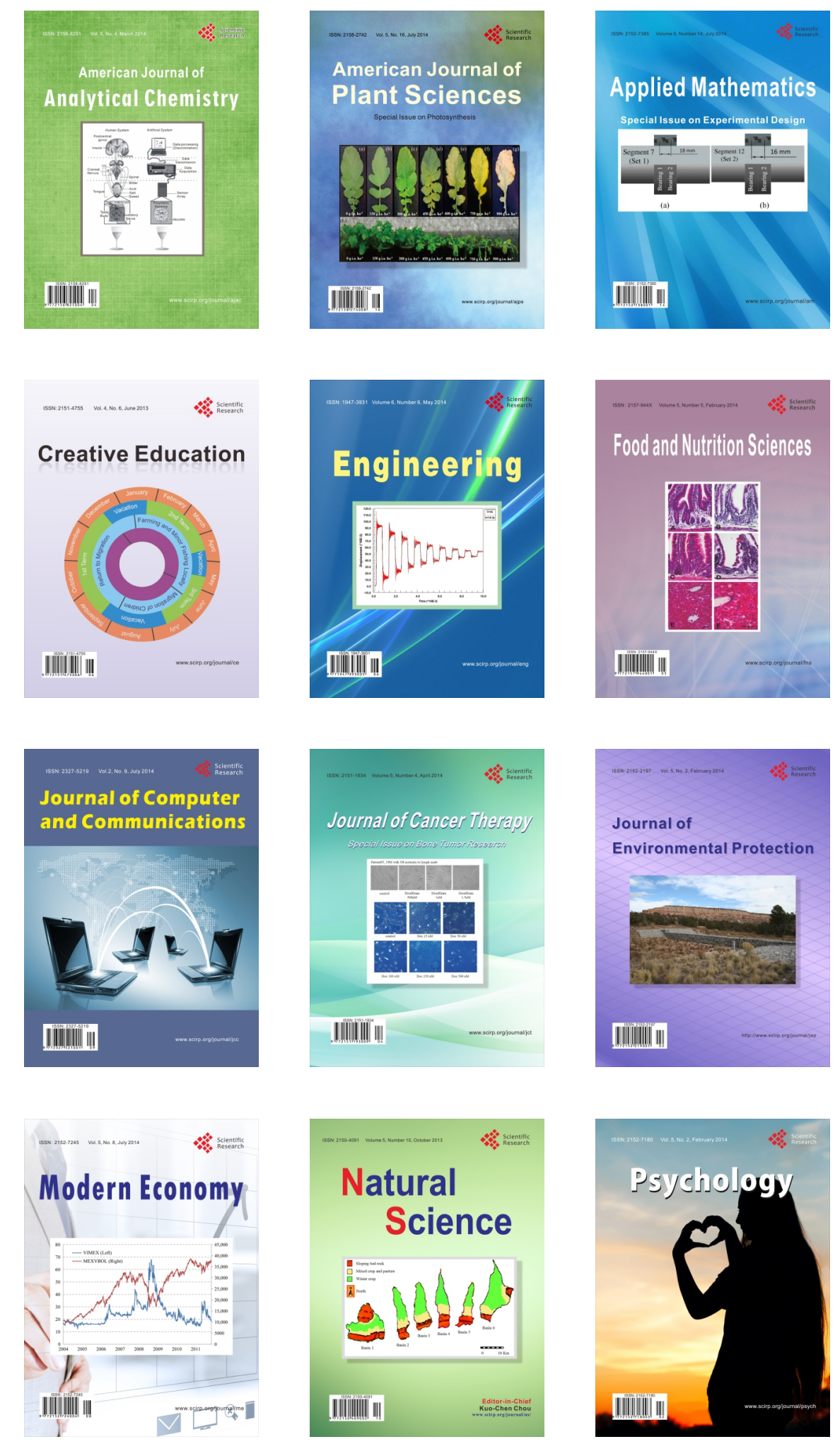